\title{
Effects of Trends and Seasonalities on Robustness of the Hurst Parameter Estimators
}

\author{
Xianming Ye ${ }^{\mathrm{a}, *}$, Xiaohua Xia ${ }^{\mathrm{a}}$, Jiangfeng Zhang ${ }^{\mathrm{a}}$, Yangquan Chen $^{\mathrm{b}, 1}$ \\ ${ }^{a}$ Department of Electrical, Electronic and Computer Engineering, University of Pretoria, Pretoria, 0002, South Africa \\ ${ }^{b}$ MESA Lab, School of Engineering, University of California, Merced, 5200 North Lake Rd., Merced, CA 95343
}

\begin{abstract}
Long-range dependence (LRD) is discovered in time series arising from different fields, especially in network traffic and econometrics. Detecting the presence and the intensity of LRD plays a crucial role in time series analysis and fractional system identification. The existence of LRD is usually indicated by the Hurst parameters. Up to now, many Hurst parameter estimators have been proposed in order to identify the LRD property involved in a time series. Since different estimators have different accuracy and robustness performances, in this paper, 13 most popular Hurst parameter estimators are summarised and their estimation performances are investigated. LRD processes with known Hurst parameters are generated as the control data set for the robustness evaluation. In addition, three types of LRD processes are also obtained as the test signals by adding noises in terms of means, trends and seasonalities to the control data set. All 13 Hurst parameter estimators are applied to these LRD processes to estimate the existing Hurst parameters. The estimation results are documented and quantified by the standard errors. Conclusions of the accuracy and robustness performances of the estimators are drawn by comparing the estimation results.
\end{abstract}

Keywords: LRD, Hurst parameters, fGn, robustness.

\section{Introduction}

The study of long-range dependence (LRD) has received considerable attention in diverse research areas, such as agronomy, astronomy, chemistry, economics, engineering, environmental science, geoscience, hydrology, mathematics, physics and statistics [1]. The LRD phenomenon is known as the dependence between observations far away in time. The presence and intensity of LRD are traditionally measured by the Hurst parameters, $H$, introduced by Hurst [2] during his studies on Nile discharges and problems related to water storage. The $H$ parameter ranges in $(0,1)$. From a physical point of view, $H$ is a measure of roughness; the roughness or anti-correlation in the signal is maximal when $H$ is close to zero. White noise with zero correlation has $H=0.5$. Smoother correlated signals have $H$ near 1.0 [3].

The Hurst parameter has a close relationship with power law, long memory, fractal, fractional calculus and chaos theory. Detection of LRD is crucial to time series analysis, especially to fractional system identification and prediction [4]. Many methods for estimating $H$ are proposed in the literature. For instance, the oldest and most common method is the Re-scaled Range (R/S) method [5]. The Aggregated Variance method [6] is based on a dispersional analysis. The Periodogram method [7] is the linear regression of the log periodogram. The Whittle estimator [8] is obtained by minimising the objective function based on the periodogram. Abry and Veitch's method [9]

\footnotetext{
*Corresponding author. Tel.: +27 (0)12 420 4353; fax: +27 (0)12 3625000 Email address: xianming.ye@up.ac.za (Xianming Ye)

${ }^{1}$ Yangquan Chen's affiliation before 2012: Electrical and Computer Engineering Department, Utah State University, 4120 Old Main Hill, Logan, UT84322, USA
}

is a wavelet-based analysis tool of $H$. Higuchi's method [10] is based on fractal theory. Most of the above-mentioned estimators are based on linear regression with graphical analysis, except the Whittle estimator.

Different estimators have different accuracy and robustness performances. Previous studies, [4], [6], [8] and [11], have made intensive efforts to compare the accuracy and robustness of the existing Hurst parameter estimators. In [4], 12 Hurst parameter estimators are analysed to compare their robustness against three kinds of noises, namely the $30 \mathrm{~dB}$ signalto-noise ratio (SNR) white Gaussian noise, $30 \mathrm{~dB}$ SNR stable noise and Fractional Autoregressive Integrated Moving Average (FARIMA) with stable innovations. In [6], Taqqu and his co-workers summarise nine Hurst parameter estimators and compare their performances when they are applied to both the fractional Gaussian noise (fGn) and $F A R I M A(0, d, 0)$ processes at some determined Hurst parameter values. Reference [8] concentrates on comparing the robustness of the Whittle-type estimators; both the Gaussian innovations and the infinite variance symmetric stable innovations are considered. In [11], the robustness of Hurst parameter estimators for noisy multifractional processes, and multifractional processes with infinite secondorder statistics is tested and analysed.

However, none of these studies clearly provides sufficient information for selecting the most suitable Hurst parameter estimators to detect the LRD properties involved in a time series with the presence of means, trends and seasonalities. In this study, 4 different LRD processes, namely, LRD process with known Hurst parameters, LRD process with non-zero means, LRD process with linear trends, and LRD process with seasonalities are generated to evaluate the robustness of existing 
Hurst parameter estimators. In the following, 13 most popular existing Hurst parameter estimators are documented and investigated, (i) Re-scaled Range (R/S) method [5]; (ii) Aggregated Variance method [12]; (iii) Difference Variance method [6]; (iv) Absolute Value method [5]; (v) Variance of Residuals method [6]; (vi) Periodogram method [7]; (vii) Modified Periodogram method [13]; (viii) Whittle estimator [8]; (ix) Diffusion Entropy method [14]; (x) Kettani and Gubner's method [15]; (xi) Abry and Veitch's method [9]; (xii) Koutsoyiannis' method [16]; (xiii) Higuchi's method [10]. A brief summary for all the above-mentioned 13 Hurst parameter estimators can be found in [4]. These estimators are applied to the 4 LRD processes. The estimation results are quantified by the standard errors. The robustness evaluation results show that the presence of trends and seasonalities has an essential influence on most of the 13 Hurst parameter estimators. However, Abry and Veitch's method exhibits strong robustness to the trends, whereas the Whittle estimator is not vulnerable to seasonalities.

The paper is organised as follows: in Section 2, preliminary studies on LRD property and fGn processes are reviewed. In Section 3, the test signals are generated and Hurst parameter estimator evaluation procedures are provided. Subsequently, the performances of the 13 estimators against means, trends and seasonalities are investigated and the results are given in both graphic styles and quantified by the standard errors in Section 4. The performances of the 13 estimators are summarised in Section 5 .

\section{Preliminaries}

Preliminaries on LRD and fGn are essential for this study. Detailed descriptions of these two concepts are provided below.

\subsection{Long-range dependence}

A stationary process with finite second-order statistics is said to have long-range dependence if its covariance function $C(n)$ decays slowly as $n \rightarrow \infty$. That is, there exists an $\alpha, 0<\alpha<1$, such that

$$
\lim _{n \rightarrow \infty} \frac{C(n)}{n^{-\alpha}}=c
$$

where $c$ is a finite, positive constant. That is to say, for large $n$, $C(n)$ is similar to $c / n^{\alpha}$ [17]. The parameter $\alpha$ has a relationship to $H$ as $\alpha=2-2 H$. The LRD can also be defined by the spectral density. A weak stationary time series $X_{i}$ is said to be long-range dependent if its spectral density follows

$$
f(\lambda) \sim C_{f}|\lambda|^{-\beta},
$$

as $\lambda \rightarrow \infty$, for certain $C_{f}>0$ and real parameter $\beta \in(0,1)$. The parameter $\beta$ is related to the Hurst parameter by $H=(1+\beta) / 2$ [18]. For $0.5<H<1$ the process has long-range dependence, for $H=0.5$ the observations are uncorrelated, and for $0<H<$ 0.5 the process has short-range dependence and the correlations sum up to zero.

\subsection{Fractional Gaussian noise}

Before testing the Hurst parameter estimators, some control data set with known Hurst parameters are reqired. The control data are better synthesised from the first principle of fractional Brownian motion (fBm), which is a Gaussian process as defined in [12]. Successive increments of an fBm are called fGn [19], which is defined as follows.

Let $X_{i}$ denote a time series. Then $X_{i}$ is second-order stationary if its mean value $E\left(X_{i}\right)$ does not depend on $i$ and if the auto-covariance function $E\left[\left(X_{i}-E\left(X_{i}\right)\right)\left(X_{j}-E\left(X_{j}\right)\right)\right]$ depends on $i$ and $j$ only through their difference $k=i-j$, in which case one has

$$
\gamma(k)=E\left[\left(X_{i}-E\left(X_{i}\right)\right)\left(X_{i-k}-E\left(X_{i-k}\right)\right)\right] .
$$

The variance of the process is $\sigma^{2}=\gamma(0)=E\left[\left(X_{i}-E\left(X_{i}\right)\right)^{2}\right]$, and the autocorrelation function is $\rho(k)=\gamma(k) / \sigma^{2}$. A second-order stationary process is said to be exactly second-order self-similar with Hurst exponent $H \in(0,1)$ if

$$
\gamma(k)=\left(\sigma^{2} / 2\right)\left(|k+1|^{2 H}-2|k|^{2 H}+|k-1|^{2 H}\right),
$$

or equivalently

$$
\rho(k)=\frac{1}{2}\left(|k+1|^{2 H}-2|k|^{2 H}+|k-1|^{2 H}\right) .
$$

If $X_{i}$ is a Gaussian process, then it is known as an fGn.

\section{Test signal generation and Hurst parameter estimator evaluation procedures}

\subsection{Test signal generation}

In order to evaluate the robustness of the 13 Hurst parameter estimators against means, trends and seasonalities, test signals that exhibit the LRD properties with known Hurst parameters are required as control data sets. For this purpose, fGn time series, denoted by $F_{k}\left(t, H_{j}\right)$ with the standard deviation $\sigma=1$ and known Hurst parameters $H_{j}$ are adopted to generate the test signals. Four LRD processes are produced for the robustness evaluation for the 13 estimators. As shown in Equation (6),

$$
\tilde{F}_{k}\left(t, \hat{H}_{j}\right)=F_{k}\left(t, H_{j}\right)+a+b t+c \sin (2 \pi f t),
$$

$\hat{H}_{j}$ denotes the Hurst parameters that need to be characterised from the following 4 LRD processes that are produced by adjusting the coefficients $a, b$ and $c$ in (6).

1) LRD 1: LRD processes with known Hurst parameters, $\tilde{F}_{k}\left(t, \hat{H}_{j}\right)=F_{k}\left(t, H_{j}\right)$;

2) LRD 2: LRD processes with non-zero means, $\tilde{F}_{k}\left(t, \hat{H}_{j}\right)=$ $F_{k}\left(t, H_{j}\right)+a$

3) LRD 3: LRD processes with linear trends, $\tilde{F}_{k}\left(t, \hat{H}_{j}\right)=$ $F_{k}\left(t, H_{j}\right)+b t$

4) LRD 4: LRD processes with seasonalities, $\tilde{F}_{k}\left(t, \hat{H}_{j}\right)=F_{k}\left(t, H_{j}\right)+c \sin (2 \pi f t)$. 
$H_{j}$ increases from 0.01 to 0.99 in steps of $0.01, j=1,2, \ldots, 99$; for the estimator evaluation, each of the 4 LRD processes will be generated 100 times for every $H_{j}$, where $k=1,2, \ldots, 100$ represents the $k$-th generated LRD process; $t=1,2, \ldots, 8760$ since the length of an hourly sampled time series for 365 days in a year is $365 \times 24=8760$. LRD 1 represents the LRD processes with known Hurst parameters, LRD 2-4 are the LRD processes with additive noises in terms of means, trends and seasonalities, respectively. It is noted that during the evaluation process of the 13 estimators, the mean, trend and seasonality noises will only be added separately.

\subsection{Hurst parameter estimator evaluation procedures}

In this section, detailed Hurst parameter estimator robustness evaluation procedures are provided as shown in the flow chart in Fig. 1. For LRD 1, the Hurst parameters are estimated by different Hurst parameter estimators by the following steps:

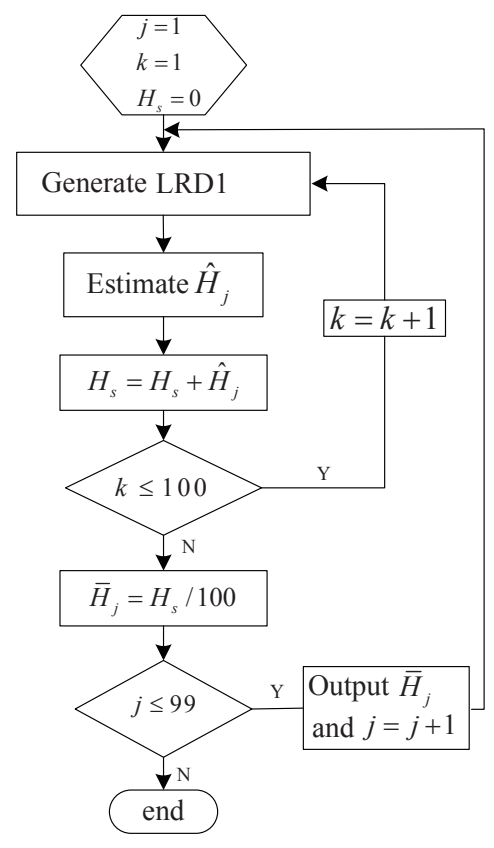

Figure 1: Flow chart of the Hurst parameter estimator evaluation

Step 1: Let $j=1, k=1$ and the temporary variable $H_{s}=0$.

Step 2: In the inner loop of the flow chart, 100 replications of each of the 4 LRD processes are generated. For the $k$-th generated LRD process, $\hat{H}_{j}$ is estimated by a certain estimator, and then summed up and stored in $H_{s}$.

Step 3: The final estimated Hurst parameter $\bar{H}_{j}$ is the average of the 100 estimates of $\hat{H}_{j}$.

Step 4: For the outer loop, Step 2 and 3 will repeat 99 times until all $\bar{H}_{j}$ 's are obtained.

During the robust evaluation for each Hurst parameter estimator, $\hat{H}_{j}$ of LRD 1 is taken as a benchmark for comparison purpose. By following similar evaluation procedures, the final estimated Hurst parameters of LRD 2-4 can also be obtained. The
Table 1: Initial coefficients for the evaluation

\begin{tabular}{ll}
\hline LRD processes & Coefficients in Eq. (6) \\
\hline \hline LRD 1 & $a=0, b=0, c=0$ \\
\hline \multirow{2}{*}{ LRD 2 } & $\begin{array}{l}a=\frac{1}{N} \sum_{k=1}^{N}\left[\max \left(F_{k}\right)-\min \left(F_{k}\right)\right], \\
b=0, c=0\end{array}$ \\
\hline LRD 3 & $\begin{array}{l}a=0, c=0, \\
b=\frac{1}{N \times 8760} \sum_{k=1}^{N}\left[\max \left(F_{k}\right)-\min \left(F_{k}\right)\right],\end{array}$ \\
\hline \multirow{2}{*}{ LRD 4 } & $\begin{array}{l}a=0, b=0, f=1 / 24, \\
c\end{array}$ \\
\hline
\end{tabular}

impact of means, trends and seasonalities to the 13 estimators can be characterised by comparing the final Hurst parameter estimates between LRD 1 and LRD 2-4. For LRD 4, daily seasonalities with frequency $f=1 / 24$ are generated to add into the LRD 1 processes for the robustness evaluation of the 13 estimators. In order to achieve a fair comparison of the estimation performances for the 13 Hurst parameter estimators, the expectations of $a, b$ and $c$ for LRD 2-4 are calculated respectively as provided in Table 1, where $N$ is suggested to be a sufficient large number, say $1000000 ; F_{k}$ is short for $F_{k}(t, 0.5)$.

\section{Robustness assessment and comparison}

In this section, the robustness evaluation results of the 13 Hurst parameter estimators are provided from Fig. 2 to Fig. 14. In these figures, the solid lines (in blue) are the known Hurst parameter values $H_{j}$ as the reference lines. In order to indicate the severity of the estimation bias, two bias threshold $H_{j} \pm 0.03$, denoted by solid lines (in green) are also plotted in all these figures. The estimated Hurst parameters $\hat{H}_{j}$ of different LRD processes are denoted by lines with different styles and colours. Specifically, the estimates of LRD 1-4 are represented by a dotted line (in red), a circle line (in cyan), a dash-dotted line (in magenta) and a dashed line (in black), respectively.

\subsection{Re-scaled Range method}

The estimates by the Re-scaled Range (R/S) method for the LRD 1-4 processes are presented in Fig. 2. The dotted line (in red) and the circle line (in cyan) coincide with each other, which shows that the mean value does not severely affect the $\mathrm{R} / \mathrm{S}$ estimator. However, the estimates for LRD 1-2 are biased for almost all Hurst parameter values $(0<H<1)$. $\hat{H}$ is apparently overestimated at around $H \in(0,0.6]$ and underestimated at around $H \in[0.8,1)$. In addition, the dash-dotted line (in magenta) shows that the estimated Hurst parameters are severely overestimated. Nevertheless, the dashed line (in black) is severely underestimated. It indicates that the R/S method is robust neither to the trends nor to the seasonalities.

\subsection{Aggregated Variance method}

The estimates by the Aggregated Variance method for the LRD 1-4 processes are presented in Fig. 3. The overlap of the dotted line (in red) and the circle line (in cyan) shows that the mean value does not influence the Aggregated Variance estimator. $\hat{H}$ of LRD $1-2$ is slightly biased at around $H \in(0,0.7)$. But an underestimation appears at around $H \in[0.7,1)$. However, 
the dash-dotted line (in magenta) is generally close to 1.0 and the dashed line (in black) is far underestimated. It shows that this estimator has poor robustness performance to LRD processes with trends and seasonalities.

\subsection{Difference Variance method}

The estimates by the Difference Variance method for the LRD 1-4 processes are presented in Fig. 4. The Hurst estimates for LRD 1-2 are generally underestimated and overlap with the lower bound of the bias threshold in the range $0<H<1$. Therefore, the mean value has no influence on this estimator. However, as shown by the dash-dotted line (in magenta), the estimates are obviously overestimated at around $H \in(0,0.6) \bigcap(0.9,1)$. On the contrary, the estimates are underestimated at around $H \in[0.6,0.9]$. Moreover, as shown by the dashed line (in black), the seasonalities impose a greater impact on this estimator, and $\hat{H}$ is extremely biased. Thus, the Difference Variance method is severely affected by trends and seasonalities.

\subsection{Absolute Value method}

The estimates by the Absolute Value method for the LRD 1-4 are presented in Fig. 5. The estimation performance of the Absolute Value method is very similar to the Aggregated Variance method. The estimates are nearly unbiased when $H$ is around $(0,0.7)$ but underestimated when $H$ is around $[0.7,1)$ for both LRD 1-2. The dash-dotted line (in magenta) is severely overestimated and the dashed line (in black) is always under the reference line. Thus the Absolute Value method exhibits poor estimation performance to trends and seasonalities.

\subsection{Variance of Residuals method}

The estimates by the Variance of Residuals method for the LRD 1-4 are presented in Fig. 6. The dotted line (in red) and the circle line (in cyan) show that the Variance of Residuals method estimates the Hurst parameters for both LRD 1-2 accurately given that all estimates lie within the range of the $H \pm 0.03$

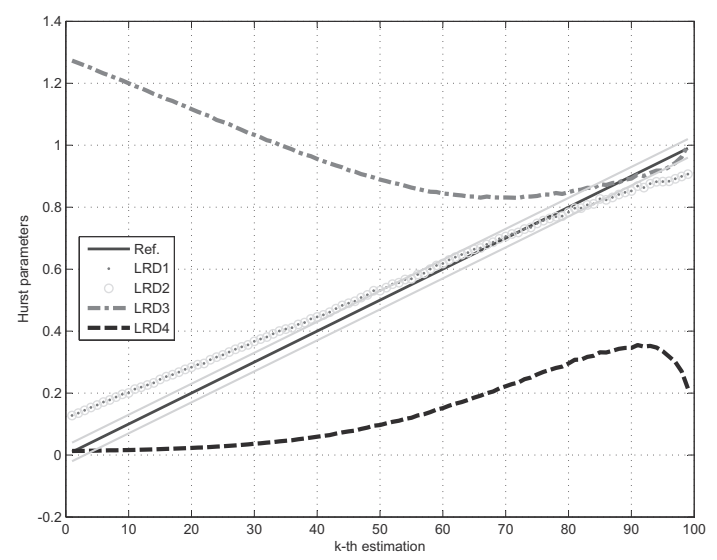

Figure 2: Estimates of LRD 1-4 by R/S method

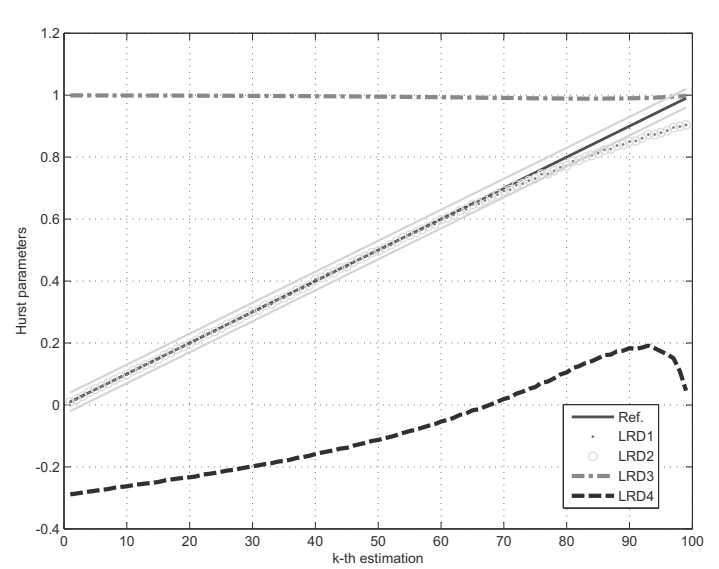

Figure 3: Estimates of LRD 1-4 by Aggregate Variance method

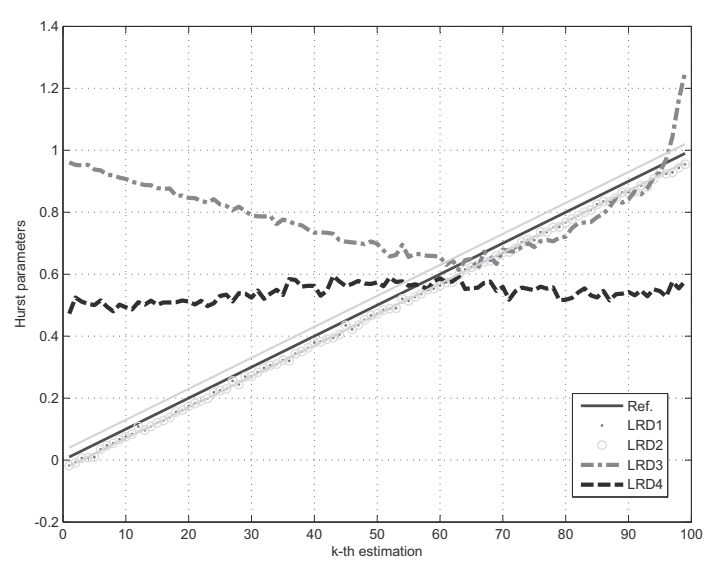

Figure 4: Estimates of LRD 1-4 by Difference Variance method

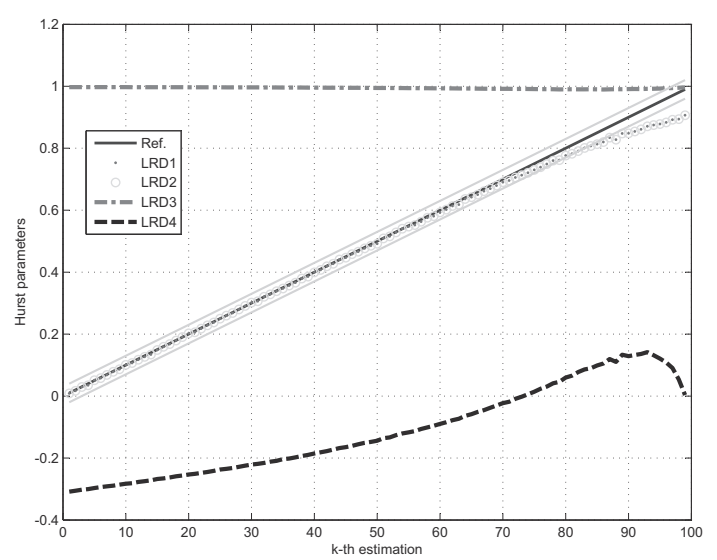

Figure 5: Estimates of LRD 1-4 by Absolute Value method 
bias thresholds (solid line in green). However, the trends influence this estimator since overestimation occurs at around $H \in(0,0.8)$ as shown by the dash-dotted line (in magenta), while only slightly biased estimation appears when $H$ is around $[0.8,1)$. The influence of the seasonalities is more severe, as shown by the dashed line (in black). The estimator can hardly give accurate estimates of LRD 4.

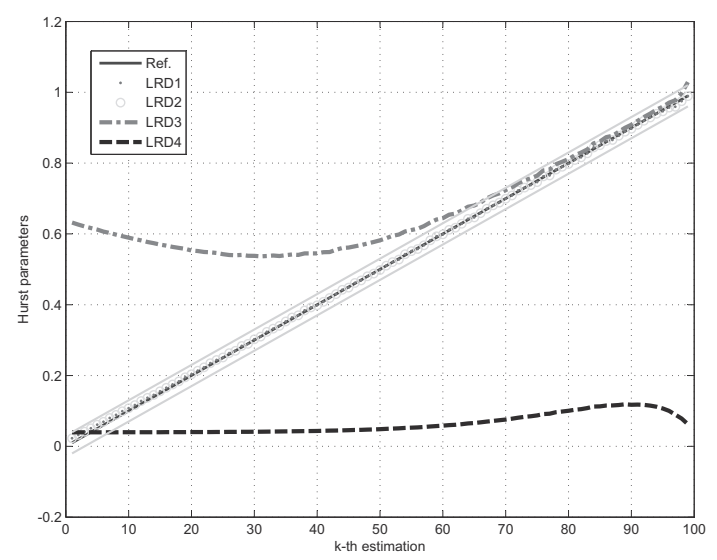

Figure 6: Estimates of LRD 1-4 by Variance of Residuals method

\subsection{Periodogram method}

The estimates by the Periodogram method for the LRD 1-4 are presented in Fig. 7. The overlap of the dotted line (in red) and the circle line (in cyan) shows that the mean value does not affect this estimator. The estimates are slightly biased when $H$ is around $(0,0.2)$ for LRD 1-2. However, the linear trends make the estimation performance poor. As shown by the dash-dotted line (in magenta), the Hurst parameters are severely overestimated. In addition, it can be observed that the seasonalities do not affect this estimator. The estimates denoted by the dashed line (in black) are slightly biased when $H$ is around $(0.2,1)$, only a little underestimated when $H$ is around $(0,0.2]$.

\subsection{Modified Periodogram method}

The estimates by the Modified Periodogram method for the LRD 1-4 are presented in Fig. 8. The dotted line (in red) and the circle line (in cyan) show that $\hat{H}$ of LRD 1-2 are a little underestimated at around $H \in(0.2,1)$ since the estimates are very close to the lower bound of the bias threshold. But the underestimation of $\hat{H}$ is more severe when $H$ is around $(0,0.2]$ given that the estimates are out of the range of the bias thresholds. The estimates shown by the dash-dotted line (in magenta) is severely overestimated. In addition, the estimates denoted by the dashed line (in black) is consistently underestimated when $0<H<1$. Generally, the modified Periodogram method is severely influenced by the linear trends.

\subsection{Whittle estimator}

The estimates by the Whittle estimator for the LRD 1-4 are presented in Fig. 9. From the dotted line (in red) and the circle line (in cyan), it is found that the estimation of $H$ is slightly biased and only a little underestimated when $H$ is close to zero. It indicates that the Whittle estimator is robust to the means. As shown by the dash-dotted line (in magenta), the trends severely affect the Whittle estimator. The estimated $\hat{H}$ is overestimated and generally greater than 1.0. The dashed line (in black) shows that seasonalities have little influence on the Whittle estimator since the estimates are generally within the range of the bias thresholds.

\subsection{Diffusion Entropy method}

The estimates by the Diffusion Entropy method for the LRD 1-4 are presented in Fig. 10. The dotted line (in red) and the circle line (in cyan) show that the estimates are slightly biased when $H$ is around $(0,0.7)$, and a little underestimated when $H$ is around $[0.7,1.0)$. The mean value does not severely influence the Diffusion Entropy method. However, the estimates $\hat{H}$ are always in range $(0.8,1.0)$ as provided by the dash-dotted line (in magenta). Thus the Diffusion Entropy estimator is generally overestimated with respect to the impact of linear trends. In addition, the dashed line (in black) shows that the estimates for LRD 4 are severely underestimated.

\subsection{Kettani and Gubner's method}

The estimates by Kettani and Gubner's method for the LRD 1-4 are presented in Fig. 11. The estimates for LRD 1 are generally accurate when $H$ is around $(0,0.9)$ and only a little underestimated at around $H \in[0.9,1)$. The mean value does not affect Kettani and Gubner's method. Thus the dotted line (in red) and the circle line (in cyan) coincide with each other. However, from the dash-dotted line (in magenta) and the dashed line (in black), it is clear that the estimates of LRD 3-4 are severely overestimated.

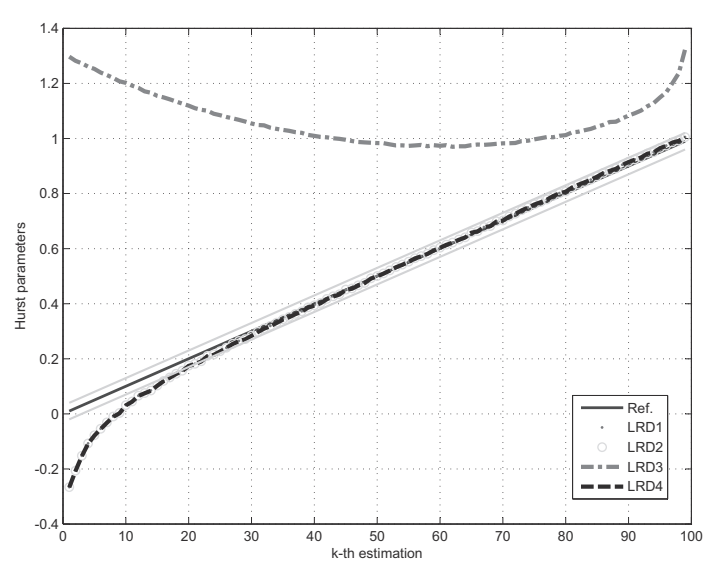

Figure 7: Estimates of LRD 1-4 by Periodogram method 


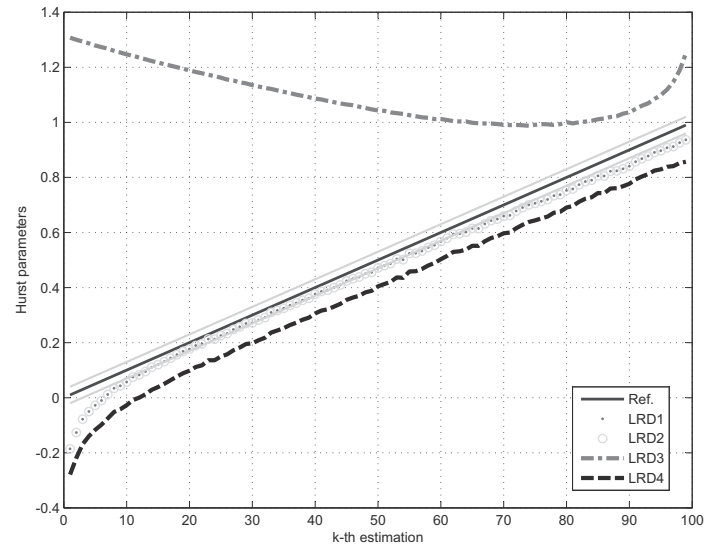

Figure 8: Estimates of LRD 1-4 by Modified Periodogram method

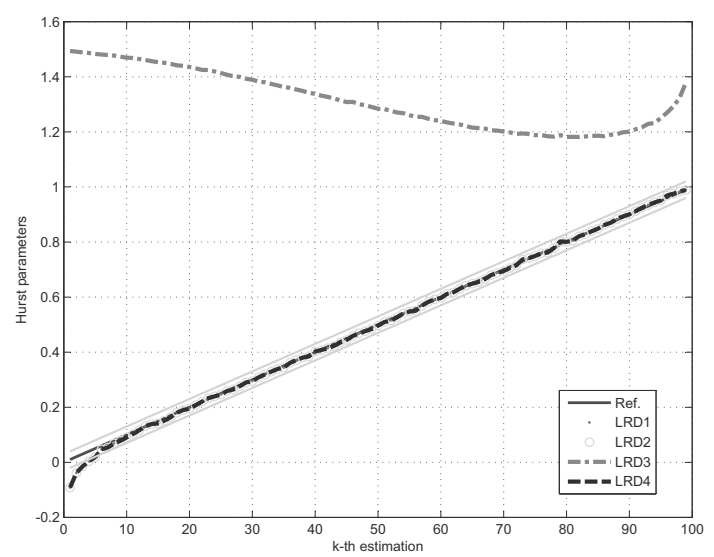

Figure 9: Estimates of LRD 1-4 by Whittle estimator

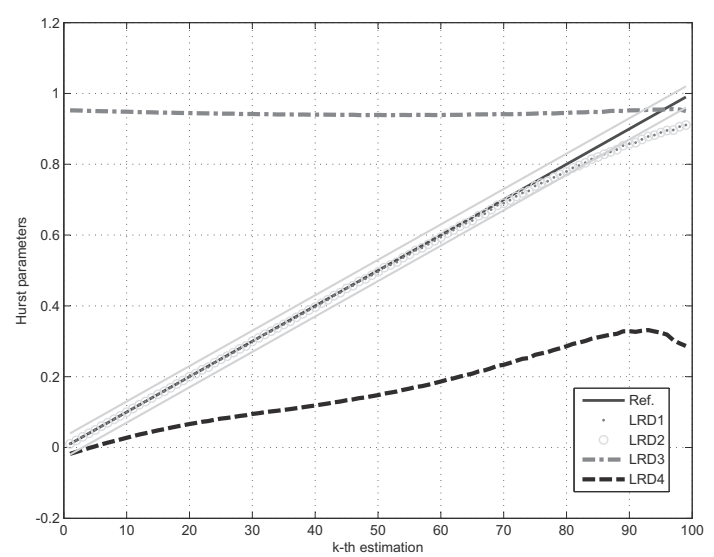

Figure 10: Estimates of LRD 1-4 by Diffusion Entropy method

\subsection{Abry and Veitch's method}

The estimates by Abry and Veitch's method for the LRD 14 processes are presented in Fig. 12. The Abry and Veitch's method is a wavelet-based Hurst parameter estimator. In this study, the Daubechies wavelet is chosen as the mother wavelet and the number of vanishing moments is 3 for the estimator evaluation. More detailed explanation can be found in [9]. It is very interesting to see that three of the estimation curves for LRD 1-3 overlap in Fig. 12. $\hat{H}$ is slightly biased when $H$ is around $(0.1,1.0)$, and underestimated at around $H \in(0,0.1]$. The results indicate that this estimator is robust to the means and trends. However, the dashed line (in black) is severely underestimated. It is evident that Abry and Veitch's method is poor in estimating the Hurst parameters from time series with seasonalities.

\subsection{Koutsoyiannis' method}

The estimates by Koutsoyiannis' method for the LRD 1-4 processes are presented in Fig. 13. From the dotted line (in red) and the circle line (in cyan), it is found that the estimates of LRD 1-2 are generally accurate at around $H \in(0.1,0.9)$. However, this estimator tends to be infinite when $H$ is close to 1.0 as can be found from the dotted line and the circle line. The reason for the infinity estimates are given in [16] and [20], where detailed numerical method in finding $\hat{H}$ is also provided in the two references. In addition, the estimator is very sensitive to the linear trends given that the all estimates for LRD 3 are tends to be infinite. From the dashed line (in black) we find that the estimates are in the range $(0.6,0.7)$ due to the impact of the seasonalities.

\subsection{Higuchi's method}

The estimates by Higuchi's method for the LRD 1-4 processes are presented in Fig. 14. The dotted line (in red) shows that the estimates are generally accurate for all $H \in(0,1)$. But this estimator is severely vulnerable to the means, trends and seasonalities. The Hurst estimation is constantly equal to 1.0

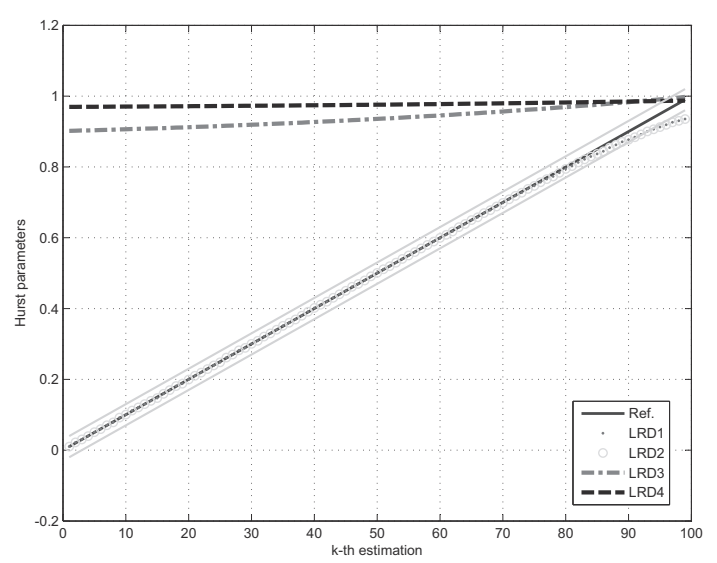

Figure 11: Estimates of LRD 1-4 by Kettani and Gubner's method 


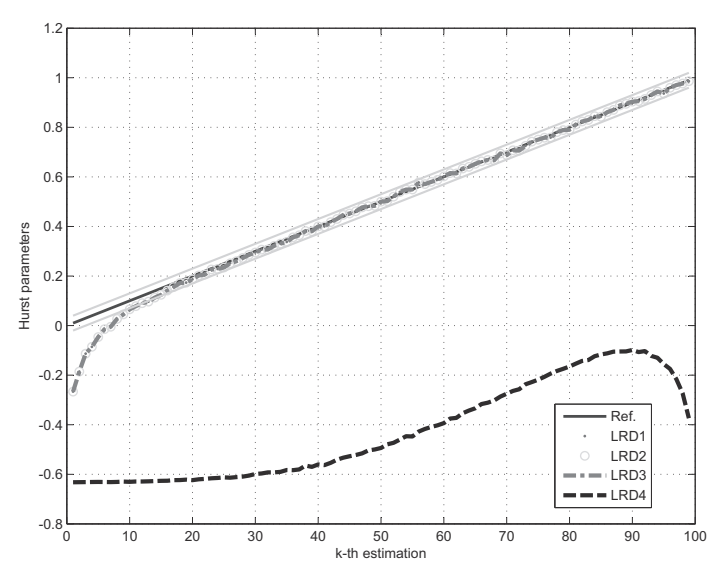

Figure 12: Estimates of LRD 1-4 by Abry and Veitch's method

for LRD 2-3. On the other hand, the estimates are generally underestimated in LRD 4.

\subsection{Quantitative comparison of the estimation results}

From Fig. 2 to Fig. 14 we can roughly compare the robustness performances of the 13 Hurst parameter estimators. In order to quantify the robustness, the standard errors $S$ of different estimations are calculated. $S$ is defined as

$$
S=\sqrt{\frac{\sum_{j=1}^{n}\left(H_{j}-\bar{H}_{j}\right)^{2}}{n-1},}
$$

where $n$ is the number of estimated Hurst parameters by each estimator and $n=99$ in this study. Table 2 gives the standard errors of the estimates for the 4 different LRDs.

In Table 2, we can find that the standard errors for LRD 1-2 are generally smaller than the standard errors for LRD 3-4. It indicates that the linear trends and seasonalities tend to impose worse effects to the Hurst parameter estimators than the mean

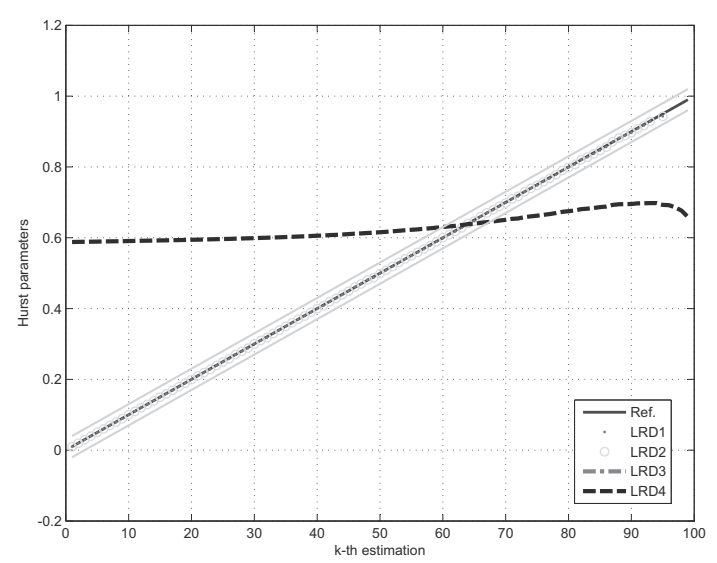

Figure 13: Estimates of LRD 1-4 by Koutsoyiannis' method

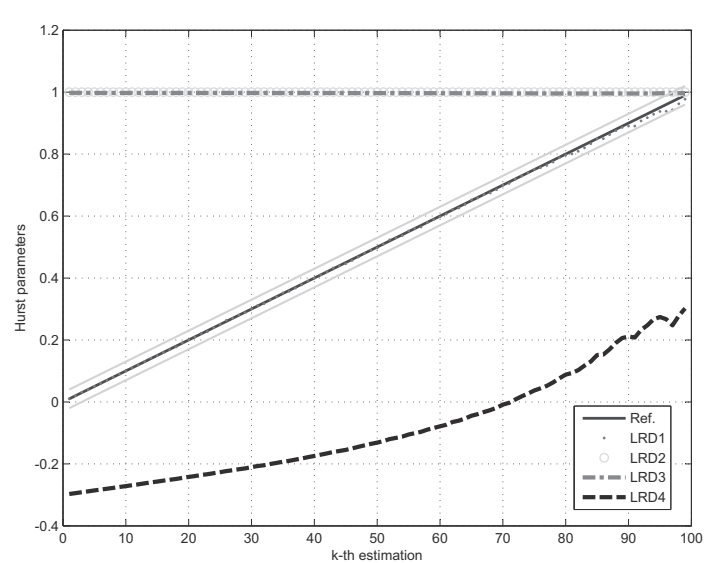

Figure 14: Estimates of LRD 1-4 by Higuchi's method

Table 2: Standard errors of Hurst parameter estimates

\begin{tabular}{l|cccc}
\hline Estimators & LRD 1 & LRD 2 & LRD 3 & LRD 4 \\
\hline \hline R/S & 0.0613 & 0.0613 & 0.6252 & 0.4038 \\
Aggregated Variance & 0.0258 & 0.0258 & 0.5758 & 0.5995 \\
Difference Variance & 0.0311 & 0.0311 & 0.4395 & 0.2771 \\
Absolute Value & 0.0261 & 0.0261 & 0.5750 & 0.6347 \\
Variance of Residuals & 0.0045 & 0.0045 & 0.2514 & 0.5124 \\
Periodogram & 0.0529 & 0.0529 & 0.6610 & 0.0532 \\
Modified Periodogram & 0.0483 & 0.0483 & 0.7021 & 0.1164 \\
Whittle estimator & 0.0144 & 0.0144 & 0.9040 & 0.0144 \\
Diffusion Entropy & 0.0224 & 0.0224 & 0.5307 & 0.3855 \\
Kettani and Gubner & 0.0129 & 0.0129 & 0.5131 & 0.5560 \\
Abry and Veitch & 0.0441 & 0.0441 & 0.0441 & 0.9407 \\
Koutsoyiannis & N/A & N/A & N/A & 0.2838 \\
Higuchi & 0.0062 & 0.5788 & 0.5764 & 0.6006 \\
\hline
\end{tabular}

offset. It is also interesting to find that the standard errors for LRD 1-2 are the same except for the Higuchi's method, which indicates that the mean values generally have no influence to 12 of the estimators. In addition, we can also find the best estimator for LRD 1-4 by looking up the minimum standard errors in each column. For instance, the Variance of Residuals method is the best one for LRD 1-2, Abry and Veitch's method is the most suitable estimator for LRD 3 while the Whittle estimator is recommended to estimate Hurst parameter from LRD 4. However, it is also clear that none of the estimators consistently performed a good estimation performance across 4 different LRD processes.

\section{Conclusion and discussion}

In this study, the robustness performances of the 13 Hurst parameter estimators have been evaluated for different LRD processes with the existence of non-zero means, linear trends and seasonalities. Since only a certain level of the mean noise and linear trend are added into LRD 1 and not all frequencies of the seasonalities have been tested in this study, the conclusion might not be decisive. However, the robustness assessment pro- 
cedures developed in this paper are applicable to practical scenarios, such as the prices in stock markets, residential daily energy usage, etc., where means, trends and daily seasonalities have been exhibited. In addition, the robust evaluation results offer useful information in choosing the most appropriate Hurst parameter estimator for a particular LRD process in order to avoid misusing of these estimators. For instance, for an LRD process with mean offset, the Variance of Residuals method is highly recommended since the standard error by this estimator is less than $1 \%$. In addition, if an LRD process exhibits linear trends, the Abry and Veitch's method is suggested to be used to estimate the Hurst parameter for this LRD process. Moreover, given an LRD process with seasonalities, the Whittle estimator proves to be the most suitable estimator for the Hurst parameter estimation.

Besides all the above-mentioned contributions of this study, more remarkable and challenging issues related to this study are raised. These issues are listed in the following and they are going to be considered and addressed in our ongoing and future work.

Firstly, one may argue that under the real world scenario, the mean offset, the trends and seasonalities are not confined in isolation, for instance, data sampled from the stock markets, and residential daily energy usage. In this case, an alternative way of estimating the Hurst parameter is to conduct signal decomposition by separating the mean, trend, seasonality and the random component in Time Series Analysis [21]. The coefficients of mean, trend and seasonality can be obtained by curve fitting and the random component can be modeled as an fBm.

Secondly, the constant variance of the LRD processes is ideal but not realistic. To further examine the robustness of estimators for real data, it is plausible to model the variance term by the Autoregressive Conditional Heteroskedasticity (ARCH), the Generalised ARCH model, the stochastic volatility or diffusion model if it will be used in capturing the Hurst parameter in the real data scenarios such as the above-mentioned data from stock markets or residential daily energy usage.

Thirdly, some real world data that sampled from the stock markets or residential daily energy usage, either in fGn or FARIMA type may be in multifractional or multiscales, see [22] and [23]. In those cases, the effects of trends and seasonalities to the $H$ estimators are also worth investigation.

\section{Acknowledgment}

The authors would like to express our sincere appreciation to the anonymous reviewers for their valuable comments and suggestions. The authors also thank Dr. Hu Sheng for several pieces of Matlab code of the Hurst parameter estimators. This work is supported by the National Hub for the Postgraduate Programme in Energy Efficiency and Demand Side Management at the University of Pretoria.

\section{References}

1. Beran, J.: 'Statistics for Long-Memory Processes' (Chapman \& Hall/CRC, New York, 1994, 1st edn.)
2. Hurst, H.E.: 'Long-term storage capacity of reservoirs', Transactions of the American Society of Civil Engineers, 1951, 116, (3), 770-799

3. Bassingthwaighte, J.B., Raymond, G.M.: 'Evaluation of the dispersional analysis method for fractal time series', Annals of Biomedical Engineer, 1995, 23, (4), 491-505

4. Sheng, H., Chen, Y.Q., Qiu, T.: 'On the robustness of Hurst estimators', IET Signal Processing, 2011, 5, (2), 209-225

5. Sun, R., Chen, Y.Q., Li, Q.: 'The modeling and prediction of Great Salt Lake elevation time series based on ARFIMA'. Proceedings of the ASME 2007 International Design Engineering Technical Conferences \& Computers and Information in Engineering Conference 2007, Las Vegas, Nevada, USA, 2007, 1-11

6. Taqqu, M., Teverovsky, V., Villinger, W.: 'Estimators for long-range dependence: an empirical study', Fractals, 1995, 3, (4), 785-788

7. Geweke, J., Porter-Hudak, S.: 'The estimation and application of long memory time series models', Journal of Time Series Analysis, 1983, 4, 221-238

8. Taqqu, M., Teverovsky, V.: 'Robustness of whittle-type estimators for time series with long-range dependence', Stochastic Models, 1997, 13, (4), 723-757

9. Abry, P., Veitch, D.: 'Wavelet analysis of long-range-dependent traffic', IEEE Transactions on Information Theory, 1998, 44, (1), 2-15

10. Higuchi, T.: 'Approach to an irregular time series on the basis of the fractal theory', Physica D: Nonlinear Phenomena, 1988, 31, (2), 277283

11. Sheng, H., Chen, Y.Q., Qiu, T.: 'Robustness analysis of Hurst estimators for multifractional Gaussian process'. The 4th IFAC Workshop Fractional Differentiation and its Applications, 2010, Badajoz, Spain, 1-6

12. Blok, H.J.: 'On the nature of the stock market: simulations and experiments', PhD thesis, Department of Physics and Astronomy, University of British Columbia, November 2000.

13. Montanari, A., Taqqu, M.S., Teverovsky, V.: 'Estimating long-range dependence in the presence of periodicity: an empirical study', Mathematical and Computer Modelling, 1999, 29, (10-12), 217-228

14. Grigolini, P., Palatella, L., Raffaelli, G.: 'Asymmetric anomalous diffusion: an efficient way to detect memory in time series', Fractals, 2001, 9, (4), 439-449

15. Kettani, H., Gubner, J.: 'A novel approach to the estimation of the long-range dependence parameter', IEEE Transactions on Circuits and Systems, 2006, 52, (6), 463-467

16. Koutsoyiannis, D.: 'Climate change, the Hurst phenomenon, and hydrological statistics', Hydrological Science Journal, 2003, 48, (1), 3-24

17. Gubner, J.A.: 'Probability and Random Processes for Electrical and Computer Engineers' (Cambridge University Press, Cambridge, 2006, 1st edn.)

18. Clegg, R.G.: 'A practical guide to measuring the Hurst parameter', International Journal of Simulation: Systems, Science $\mathcal{E}$ Technology, 2006, 7, (2), 3-14

19. Mandelbrot, B.B., Van Ness, J.W.: 'Fractional Brownian motions, fractional noises and applications', SIAM Review, 1968, 10, (4), 422-437

20. Koutsoyiannis, D.: 'Supplementary material for the paper climate change, the Hurst phenomenon, and hydrological statistics', Technical Report, Department of Water Resources, Faculty of Civil Engineering, National Technical University, Athens, 2003, http://www.itia.ntua.gr/getfile/537/2/2003HSJHurstSuppl.pdf, accessed October 2012

21. Brockwell, P., Davis, R.: 'Introduction to Time Series and Forecasting' ( Springer, New York, 2002, 2nd edn.)

22. Li, M., Zhao, W.: 'Quantitatively investigating locally weak stationarity of modified multifractional Gaussian noise', Physica A, 2012, 391, 62686278

23. Li, M., Zhao, W.: 'Representation of a stochastic traffic bound', IEEE Transactions on Parallel and Distributed Systems, 2010, 21, (9), 13681372 